\title{
SPECTRAL APPROXIMANTS OF NORMAL OPERATORS
}

\author{
by P. R. HALMOS $\dagger$ \\ (Received 19th February 1973)
}

\section{Introduction}

For each non-empty subset $\Lambda$ of the complex plane, let $\mathscr{S}(\Lambda)$ be the set of all those operators (on a fixed Hilbert space $H$ ) whose spectrum is included in $\Lambda$. The problem of spectral approximation is to determine how closely each operator on $H$ can be approximated (in the norm) by operators in $\mathscr{S}(\Lambda)$. The problem appears to be connected with the stability theory of certain differential equations. (Consider the case in which $\Lambda$ is the right half plane.) In its general form the problem is extraordinarily difficult. Thus, for instance, even when $\Lambda$ is the singleton $\{0\}$, so that $\mathscr{S}(\Lambda)$ is the set of quasinilpotent operators, the determination of the closure of $\mathscr{S}(\Lambda)$ has been an open problem for several years (3, Problem 7).

The problem of normal spectral approximation is the one in which $\mathscr{S}(\Lambda)$ is replaced by the set $\mathscr{N}(\Lambda)$ of all those normal operators whose spectrum is included in $\Lambda$. This. problem too is important and difficult. Thus, for instance, even when $\Lambda$ is the entire complex plane, for a long time it was not known whether $\mathscr{N}(\Lambda)$ approximants always exist. In other words: is it true that, for each operator $A$, the distance $\inf \{\|A-N\|: N \in \mathcal{N}(\Lambda)\}$ is always attained? An example proving that the answer is no was recently found by $D$. D. Rogers, but, even with that new information, the visible facts are greatly outnumbered by the submerged ones.

For some special sets $\Lambda$ the theory of $\mathcal{N}(\Lambda)$ approximation is at least partly known. If, for instance, $\Lambda$ is the real line, the problem becomes that of Hermitian approximation, and, at least as far as the existence of approximants is concerned, it is solved by the mapping $A \mapsto \frac{1}{2}\left(A+A^{*}\right)$. If $\Lambda$ is the unit circle (perimeter), the problem is that of unitary approximation; this case has been studied by Fan and Hoffman (1) and van Riemsdijk (7). If $\Lambda$ is the set of non-negative real numbers, the problem is that of positive approximation, and it can be regarded as solved (4). There are, however, many quite innocentlooking sets $\Lambda$ for which almost nothing is known about $\mathcal{N}(\Lambda)$ approximation. An interesting example is the problem of projection approximation, corresponding to the case in which $\Lambda$ is the pair $\{0,1\}$.

It may be that the general problem of spectral approximation is, in some

$\dagger$ Research supported in part by grants from the National Science Foundation (U.S.A.) and the Science Research Council (U.K.). 
sense, not the right thing to ask about. A piece of disturbing evidence is that the set $\mathscr{S}(\Lambda)$ is not in general closed; witness the classical example of a sequence of quasinilpotent operators whose limit is not quasinilpotent (2, Problem 87). It is comforting to observe that the problem of normal spectral approximation rests on a sounder basis: if $\Lambda$ is a closed set of complex numbers, then $\mathscr{N}(\Lambda)$ is a closed set of operators. This comfort is a consequence of the continuity of the spectrum for normal operators; see Newburgh (6).

The main purpose of this paper is to prove a theorem about normal spectral approximation to normal operators. A special case of the theorem asserts that if $A$ is normal, then $A$ has a projection approximant, and, moreover, it has one that is a function of $A$ (and that belongs, therefore, to the second commutant of $A$ ).

In order to understand the point at issue, it is wise to consider the commutative analogue of the problem. How does one approximate a complexvalued bounded measurable function $\phi$ (modulo sets of measure zero) by a characteristic function? A reasonable answer goes as follows. Let $F$ be the function defined on the complex plane by

$$
F(z)=\left\{\begin{array}{lll}
0 & \text { if } & \operatorname{Re} z \leqq \frac{1}{2} \\
1 & \text { if } & \operatorname{Re} z>\frac{1}{2}
\end{array}\right.
$$

(In other words: $F(z)=0$ or 1 according as $z$ is nearer to 0 or to 1 . In case $z$ is equidistant from 0 and 1 , i.e., in case $\operatorname{Re} z=\frac{1}{2}$, it doesn't much matter how $F$ decides between 0 and 1 ; the choice indicated in the formal definition above is one of the two simplest ones to write down.) The function $F \circ \phi$ is a characteristic function (and a function of $\phi$ at that), and it is, clearly, as near to $\phi$ as any characteristic function can get. (Nearness is measured by the supremum norm, of course; that is the appropriate commutative version of the operator norm.)

Every normal operator can be represented as multiplication by a suitable bounded measurable function $\phi$ on a suitable $L^{2}$ space. (This statement has to be interpreted with some care for non-separable spaces, but there is no essential loss of generality in restricting attention to the separable case.) Why then doesn't the preceding paragraph solve the problem of projection approximation to normal operators? Given a normal $A$, use the $F$ defined above and write $P=F(A)$; surely the operator $P$ is a projection that is as near to $A$ as any projection can get. Or is it?

Since $P$ is a function of $A$, the functional facts described above imply that $P$ is as near to $A$ as any projection that is a function of $A$ can get. In noncommutative approximation theory, however, the competition is keener than in the commutative case. Nothing in the commutative situation inhibits the existence of a projection that is not a function of $A$ and that is nearer to $A$ than $P$ is. The whole point of this paper is to show (not for projection approximation only, but for normal spectral approximation in general) that the keener competition does not change winners to losers. 
Two comments are in order.

(1) The enlarged competition may properly enlarge the list of those tied for first place. Consider, for example, the problem of projection approximation to the matrix

$$
A=\left(\begin{array}{lll}
0 & 0 & 0 \\
0 & 1 & 0 \\
0 & 0 & 2
\end{array}\right)
$$

The functional approximation process described above yields the approximant

$$
P=\left(\begin{array}{lll}
0 & 0 & 0 \\
0 & 1 & 0 \\
0 & 0 & 1
\end{array}\right)
$$

There are, however, many other projections that are as near to $A$ as $P$ is; the only thing that is "wrong" with them is that they are not functions of $A$. An example of such a near projection is given by

$$
Q=\left(\begin{array}{ccc}
\frac{1}{2} & \frac{1}{2} & 0 \\
\frac{1}{2} & \frac{1}{2} & 0 \\
0 & 0 & 1
\end{array}\right)
$$

Note that $Q$ doesn't even commute with $A$.

(2) The solution of the problem of normal spectral approximation to normal operators does not necessarily yield a solution of the problem of general spectral approximation to normal operators. More precisely: it is not always true that if $A$ is normal, then the distance from $A$ to $\mathscr{N}(\Lambda)$ is the same as the distance from $A$ to $\mathscr{S}(\Lambda)$; the latter may be strictly smaller. An example with $\Lambda=\{0\}$ is $A=\left(\begin{array}{ll}2 & 0 \\ 0 & 0\end{array}\right)$. The unique $\mathcal{N}(\Lambda)$ approximant to $A$ is

$$
0\left(=\left(\begin{array}{ll}
0 & 0 \\
0 & 0
\end{array}\right)\right)
$$

the distance $\|A-0\|$ is 2 . From the point of view of general spectral approximation, however, 0 is not a very good nilpotent approximation to $A$. The (non-normal) nilpotent operator $Q=\left(\begin{array}{rr}1 & -1 \\ 1 & -1\end{array}\right)$ is better; the distance $\|A-Q\|$ is $\sqrt{2}$.

\section{Retraction}

If, as before, $\Lambda$ is a non-empty closed subset of the complex plane, a distance-minimizing retraction for $\Lambda$ is a function $F$ mapping the complex plane into $\Lambda$ so that

$$
|z-F(z)| \leqq|z-\lambda| \text { for all } \lambda \text { in } \Lambda \text {. }
$$

The inequality explains the term "distance-minimizing"; the fact that if $z$ is in $\Lambda$, then $|z-F(z)| \leqq 0$, so that $F(z)=z$, explains " retraction". Since 
the only retractions to be considered in the sequel are distance-minimizing ones, the modifier can (and will) be omitted with no danger of confusion. The assumptions (non-empty and closed) imply that for each such $\Lambda$ at least one retraction does exist.

The solution of the problem of projection approximation to normal operators depends on a retraction for the pair $\{0,1\}$; the general case depends, similarly, on retractions for general closed sets. If $\Lambda$ is convex, then it is easy to verify, and well known, that there is a unique retraction for $\Lambda$. It is of interest to observe that the converse is true: if $\Lambda$ is a closed subset of the plane such that there is a unique retraction for $\Lambda$, then $\Lambda$ is convex. (This converse is known as Motzkin's theorem; see (8, p. 94).)

Not much can be said about retractions in general. One easy observation that is worth recording is that every retraction is bounded on bounded sets. Proof: if $\rho(z)$ is the distance from $z$ to $\Lambda$, then

$$
|F(z)| \leqq|z-F(z)|+|z| \leqq \rho(z)+|z|
$$

for all $z$, and the majorant is continuous in $z$.

Are retractions necessarily continuous, or, failing that, does at least one continuous retraction exist for each $\Lambda$ ? The answer is no. Retractions can refuse to be continuous; if $\Lambda=\{0,1\}$, then there is no continuous retraction for $\Lambda$. It is, however, a part of the standard lore of the subject that if $\Lambda$ is convex, then the unique retraction for $\Lambda$ is necessarily continuous. In the converse direction, Motzkin's theorem says that uniqueness implies convexity; is there any sense in which continuity also implies convexity? The answer is yes: if $\Lambda$ is such that there exists a continuous retraction $F$ for $\Lambda$, then there can be only one retraction for $\Lambda$ and therefore, by Motzkin's theorem, $\Lambda$ is convex. Here is a simple proof (discovered by A. M. Davie). If some $z_{0}$ has two distinct nearest points in $\Lambda$, say $\alpha$ and $\beta$, then the open disc with centre $z_{0}$ and radius $\left|z_{0}-\alpha\right|\left(=\left|z_{0}-\beta\right|\right)$ can contain no point of $\Lambda$. It follows that each point $z$ of the open segment $\left(z_{0}, \alpha\right)$ has $\alpha$ as its unique nearest point in $\Lambda$ (i.e., $F(z)=\alpha$ ), and, similarly, each point $z$ of the open segment $\left(z_{0}, \beta\right)$ has $\beta$ as its unique nearest point in $\Lambda$ (i.e., $F(z)=\beta$ ). Since this contradicts the continuity of $F$ at $z_{0}$, the proof is complete.

Retractions may even fail to be Borel measurable. If, for instance,

$$
\Lambda=\{0,1\},
$$

then the value of a retraction for $\Lambda$ is uniquely determined whenever $\operatorname{Re} z \neq \frac{1}{2}$. If, however, $\operatorname{Re} z=\frac{1}{2}$, then there is great freedom of choice; assign to each point on that line either 0 or 1, arbitrarily, and conclude, by a familiar cardinal number argument, that it is possible to do so in a manner that is not Borel measurable. In view of this example it is pleasant to learn that, although pathology can exist, it can always be avoided. This is, in fact, the most useful single assertion about retractions: they cannot refuse to be Borel measurable. 
Lemma. If $\Lambda$ is a non-empty closed subset of the complex plane, then there exists a Borel measurable retraction for $\Lambda$.

The assertion can be made to follow from the work of Kuratowski and Ryll-Nardzewski (5); what follows is an easy special proof for the special case at hand.

For each complex number $z$, write $\rho(z)$ for the distance from $z$ to $\Lambda$, and consider the circle (perimeter) with centre $z$ and radius $\rho(z)$. (The degenerate case $\rho(z)=0$, i.e., the case in which $z$ belongs to $\Lambda$, does not require special treatment.) By definition, $\rho(z)$ is the smallest radius with the property that the circle meets $\Lambda$; the intersection of the circle with $\Lambda$ is, of course, a closed set. If $\lambda$ is in that closed set, then $\lambda-z=\rho(z) \exp i \theta$, with $0 \leqq \theta<2 \pi$; let $\theta(z)$ be the smallest value of $\theta$ (i.e. the smallest for which $\lambda$ is in $\Lambda$ ), and write

$$
F(z)=z+\rho(z) \exp i \theta(z) \text {. }
$$

That is: among the points $\lambda$ in $\Lambda$ that are at minimal distance from $z$, let $F(z)$ be the one with smallest argument as viewed from $z$.

It is to be proved that $F$ is Borel measurable; it will, in fact, be proved that $\theta$ is lower semicontinuous. Since $\rho$ is continuous, that is indeed more than enough.

Suppose $z_{n} \rightarrow z_{0}$. Write $\theta_{n}=\theta\left(z_{n}\right)$, and consider any convergent subsequence $\left\{\theta_{n_{k}}\right\}$ with $\theta_{n_{k}} \rightarrow \theta_{0}$, say. Since

$$
z_{n_{k}}+\rho\left(z_{n_{k}}\right) \exp i \theta_{n_{k}} \rightarrow z_{0}+\rho\left(z_{0}\right) \exp i \theta_{0}
$$

the point $z_{0}+\rho\left(z_{0}\right) \exp i \theta_{0}$ is in $\Lambda$; since

$$
\left|z_{0}-\left(z_{0}+\rho\left(z_{0}\right) \exp i \theta_{0}\right)\right|=\rho\left(z_{0}\right)
$$

so that $z_{0}+\rho\left(z_{0}\right) \exp i \theta_{0}$ is at minimal distance from $z_{0}$, it follows from the definition of the function $\theta$ that $\theta\left(z_{0}\right) \leqq \theta_{0}$. That is: the limit of every convergent subsequence of $\left\{\theta\left(z_{n}\right)\right\}$ is greater than or equal to $\theta\left(z_{0}\right)$, which implies that

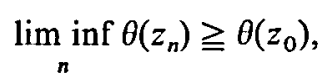

i.e., that $\theta$ is lower semicontinuous.

\section{Conclusion}

Theorem. If $F$ is a Borel measurable retraction for a non-empty closed subset $\Lambda$ of the complex plane, and if $\Lambda$ is an arbitrary normal operator, then spect $F(A) \subset \Lambda$; if $N$ is any other normal operator with spect $N \subset A$, then

$$
\|A-F(A)\| \leqq\|A-N\| .
$$

Proof. To prove the spectral assertion, use the spectral theorem. Represent $A$ as a multiplication by, say, $\phi$ on some $L^{2}$ space. Since $\operatorname{ran} F \subset \Lambda$, therefore $\operatorname{ran} F \circ \phi \subset \Lambda$. Since $\Lambda$ is closed, it follows that the essential range of $F \circ \phi$ is included in $\Lambda$ also, and the essential range of $F \circ \phi$ is exactly spect $F(A)$. 
(Note that since $F$ is bounded on bounded sets, the functional calculus is applicable, so that $F(A)$ makes sense.)

The main assertion of the theorem is the inequality. Its proof for convex $\Lambda$ is somewhat easier than for the general case, and deserves at least to be outlined.

Proof for the convex case. If $A$ has an eigenvalue $\alpha$, with an eigenvector $e$ of norm 1, so that $A e=\alpha e$, then, of course, $F(A) \mathrm{e}=F(\alpha) \mathrm{e}$. Since

$$
((A-N) e, e)=\alpha-(N e, e)=\alpha-\lambda,
$$

where $\lambda$ belongs to the numerical range $W(N)$, and since

$$
W(N)=\operatorname{conv} \text { spect } N \subset \operatorname{conv} \Lambda=\Lambda,
$$

it follows that

$$
\begin{aligned}
\|(A-F(A)) e\| & =|\alpha-F(\alpha)| \leqq|\alpha-\dot{\lambda}| \\
& =|((A-N) e, e)| \leqq w(A-N) \leqq\|A-N\| .
\end{aligned}
$$

(Here $w$ is the numerical radius.) If $A$ happens to be diagonal (i.e., if $A$ has an orthonormal basis full of eigenvectors), then the preceding conclusion implies that

$$
\|A-F(A)\| \leqq\|A-N\| \text {. }
$$

By the spectral theorem an arbitrary normal operator is the limit (in the norm) of diagonal ones. Since the continuity of $F$ implies that the mapping $X \mapsto F(X)$, defined for normal operators $X$, is continuous also, the proof in this case is complete.

Proof for the general case. Write, as before, $\rho(z)$ for the distance from $z$ to $\Lambda$.

(a) If $e$ is a unit vector, then $\rho(\alpha) \leqq\|(\alpha-N) e\|$ for every complex number $\alpha$.

For the proof, represent $N$ as multiplication by $\phi$ on $L^{2}(\mu)$. Since the spectrum of $N$ is the essential range of $\phi$, it follows that the essential range of $\phi$ is included in $\Lambda$, and therefore (2, Problem 97) $\rho(\alpha) \leqq|\alpha-\phi|$ almost everywhere. Hence

$$
\begin{aligned}
\|(\alpha-N) e\|^{2} & =\int|\alpha-\phi|^{2}|e|^{2} d \mu \\
& \geqq(\rho(\alpha))^{2} \int|e|^{2} d \mu=(\rho(\alpha))^{2} .
\end{aligned}
$$

(b) If $\alpha$ is an eigenvalue of $A$, then $\rho(\alpha) \leqq\|A-N\|$.

Indeed: if $e$ is a unit vector such that $A e=\alpha e$, then, by $(a)$,

$$
\rho(\alpha) \leqq\|(\alpha-N) e\|=\|(A-N) e\| \leqq\|A-N\| \text {. }
$$


(c) If $\alpha$ is in spect $A$, then there exists a sequence $\left\{A_{k}\right\}$ of normal operators such that $\left\|A_{k}-A\right\| \rightarrow 0$ and $\alpha$ is an eigenvalue of each $A_{k}$.

Represent $A$ as multiplication by $\phi$ on $L^{2}(\mu)$. Since $\alpha$ is in the essential range of $\phi$, it follows that if $D_{k}$ is the open disc with centre $\alpha$ and diameter $1 / k$, then $\phi^{-1}\left(D_{k}\right)$ has positive measure. Write $\phi_{k}=\alpha$ in $\phi^{-1}\left(D_{k}\right)$ and $\phi_{k}=\phi$ outside $\phi^{-1}\left(D_{k}\right)$, and let $A_{k}$ be multiplication by $\phi_{k}$. Clearly $A_{k}$ is normal and $\alpha$ is an eigenvalue of $A_{k}$ for each $k$. Since $\left|\phi_{k}-\phi\right|<1 / k$ in $\phi^{-1}\left(D_{k}\right)$ and $\left|\phi_{k}-\phi\right|=0$ outside $\phi^{-1}\left(D_{k}\right)$, it follows that $\phi_{k} \rightarrow \phi$ uniformly, and hence that $A_{k} \rightarrow A$ in the norm.

(d) If $\alpha$ is in spect $A$, then $\rho(\alpha) \leqq\|A-N\|$.

By $(c)$, there is a sequence $\left\{A_{k}\right\}$ of normal operators such that $A_{k} \rightarrow A$ and $\alpha$ is an eigenvalue of each $A_{k}$. By $(b), \rho(\alpha) \leqq\left\|A_{k}-N\right\|$ for each $k$. Since $\left\|A_{k}-N\right\| \rightarrow\|A-N\|$ as $k \rightarrow \infty$, the assertion follows.

(e) The ground is now completely prepared for the final proof.

Represent $A$ as multiplication by $\phi$ on $L^{2}$; then $A-F(A)$ is multiplication by $\phi-F \circ \phi$. Since almost every value of $\phi$ is in the spectrum of $A$, and since

$$
|\phi-F \circ \phi|=\rho \circ \phi
$$

it follows from $(d)$ that

$$
|\phi-F \circ \phi| \leqq\|A-N\|
$$

almost everywhere. The stated inequality is an immediate consequence.

A special case of the theorem (in fact, a special case of the convex special case of the theorem) appears in (4); there $\Lambda$ is the set of non-negative real numbers. The proof there is not as simple as the proof of the (more general) convex case above.

Non-convex special cases of the theorem, appearing outside the context established above, can be quite puzzling. A case in point is that of projection approximation $(\Lambda=\{0,1\})$. It is tempting in each such special case to look for a proof appropriate to it, but the search, even if successful, is not likely to throw much light on the general problem.

The theorem implies that, for each $\Lambda$, each normal oprator has an $\mathscr{N}(\Lambda)$ approximant. A direct proof of even this mild existential statement does not seem to be on the surface.

The methods used above make heavy use of the normality of the operator to be approximated. The general problem of, for instance, projection approximation to non-normal operators is still open, and not even the existence of projection approximants is known. Sometimes, however, it happens that the results for normal operators are more widely applicable than they should be. Hermitian approximation is an example (it is easy to the point of triviality for all operators, not only for normal ones), and the problems of unitary approximation and contraction approximation are also easier than one would have dared to predict. Why? 


\section{REFERENCES}

(1) K. FAN and A. J. HofFMAN, Some metric inequalities in the space of matrices, Proc. Amer. Math. Soc. 6 (1955), 111-116.

(2) P. R. Halmos, A Hilbert space problem book (Van Nostrand, Princeton, 1967).

(3) P. R. Halmos, Ten problems in Hilbert space, Bull. Amer. Math. Soc. 76 (1970), 887-933.

(4) P. R. Halmos, Positive approximants of operators, Indiana Univ. Math. J. 21 (1972), 951-960.

(5) K. Kuratowski and C. Ryll- Nardzewski, A general theorem on selectors, Bull. Acad. Polon. Sci. 13 (1965), 397-403.

(6) J. D. NewBurgh, The variation of spectra, Duke Math. J. 18 (1951), 165-176.

(7) D. J. VAN RIEMSDIJK, Some metric inequalities in the space of bounded linear operators on a separable Hilbert space, Nieuw Arch. Wisk. (3) 20 (1972), 216-230.

(8) F. A. Valentine, Convex sets (McGraw-Hill, New York, 1964).

\section{INDIANA UNIVERSITY}

AND

UNIVERSITY OF EDINBURGH 